\title{
Buffer Induced Magnetic Patterning of Ultrathin Co Layer
}

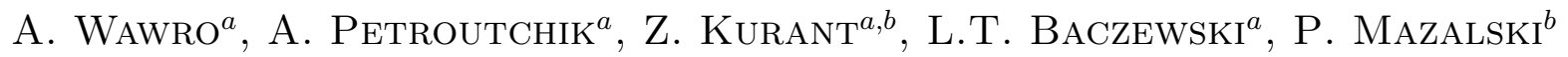 \\ AND A. MAZIEWSKI ${ }^{b}$ \\ ${ }^{a}$ Institute of Physics, Polish Academy of Sciences \\ al. Lotników 32/46, 02-668 Warsaw, Poland \\ ${ }^{b}$ Institute of Experimental Physics, University of Białystok \\ Lipowa 41, 15-424 Białystok, Poland
}

\begin{abstract}
The possibility of magnetic anisotropy engineering of ultrathin Co films in the scale of several dozen nanometers is investigated by magnetooptical magnetometry. In $\mathrm{Au} / \mathrm{Co} / \mathrm{Au}$ sandwiches the spin reorientation transition from the out-of-plane to the in-plane configuration is observed, when Co layer thickness exceeds $1.9 \mathrm{~nm}$. Molybdenum as the underlayer suppresses the Co thickness range for which the perpendicular magnetization is stable. The application of patterned buffer in the form of $\mathrm{Au}$ islands grown on Mo layer for ultrathin Co film gives rise to the array of spatially stable magnetic dots fabrication ca. $100 \mathrm{~nm}$ in lateral size with magnetization perpendicular to the film plane surrounded by the area magnetized in the sample plane.
\end{abstract}

PACS numbers: 75.70.-i, 75.30.Gw, 75.75.+a, 68.37.Ps, 78.20.Ls

\section{Introduction}

Progress in miniaturization of electronic devices and recent development of spintronics increased interest in the intentional modification of magnetic properties of various materials in nanometer scale. Patterned magnetic layered structures are promising in application as sources of very local and well defined magnetic fields or as media for magnetic recording, in which limitations originating from superparamagnetic effect are substantially reduced. Such materials are also ideal tools for basic research of nanomagnetism.

There are at least three ways to fabricate spatially patterned magnetic structures: (i) specific features of the substrate surface as reconstruction [1] or vicinal structure [2] force the growth of regular arrays of magnetic dots, (ii) spatial tailoring of properties in uniform multilayers by ion beam bombardment [3] and (iii) engineering of the magnetic anisotropy of continuous and uniform magnetic layer by spatially modified substrate [4].

In this work we report preliminary results which point for the possibilities of magnetic anisotropy engineering in Co ultrathin films in the scale of several dozen nanometers. The strong dependence of magnetic anisotropy of Co films on the type of nonmagnetic underlayer and/or overlayer in combination with an unique island-like growth of $\mathrm{Au}$ layer on Mo buffer enables the spatial switching of the Co layer magnetization between perpendicular and parallel direction to the layer plane.

\section{Experimental details}

The investigated structures were grown in molecular beam epitaxy (MBE) system equipped both with e-guns
(Mo and Co sources) and effusion cells (Au source). Sapphire monocrystalline wafers with normal axis oriented along (11-20) direction were used as the substrates. A Mo buffer layer deposited at $1000^{\circ} \mathrm{C}$ grows under such conditions in (110) direction. The optimum temperature for island-like growth of $\mathrm{Au}$ on Mo buffer was estimated for $500^{\circ} \mathrm{C}$. Ferromagnetic Co layer and Au cap layer were deposited at room temperature. The overall growth process was monitored by reflection high energy electron diffractometer (RHEED). The morphology of the buffer grown as Au islands on Mo layer was investigated by atomic force microscopy (AFM) in ambient conditions. The measurements of hysteresis loops were carried out ex situ at room temperature by means of polar Kerr magnetooptical magnetometer (P-MOKE) in the magnetic field applied in perpendicular direction to the film plane.

\section{Results and discussion}

Effective magnetic anisotropy determining the magnetization direction in ultrathin Co layers is the result of interplay of several components: shape (dipole), magnetocrystalline, magnetoelastic and interface anisotropies. The shape anisotropy always forces magnetization to align in the sample plane. The influence of the remaining components usually is not explicitly described and final result substantially depends on such factors as: the type of under- and overlayer, structure of interfaces and strains induced by the lattice mismatch of adjacent layers. In particular, such lattice mismatch at the $\mathrm{Au} / \mathrm{Co}$ interface is as large as $14 \%$, so the frequently observed coherent growth may substantially enhance the contribution of magnetoelastic component of anisotropy in the 
vicinity of the interfaces. Thus the resulting magnetization may be oriented either in parallel or perpendicularly to the sample plane.

Ultrathin Co film for numerous combinations of underand overlayer in the certain range of thickness is magnetized perpendicularly to the film plane. One of the widest ranges is observed for $\mathrm{Au} / \mathrm{Co} / \mathrm{Au}$ system and spin reorientation transition (SRT) from out-of-plane to the in-plane direction is found for Co layer thickness equal to $1.9 \mathrm{~nm}$ [5]. Replacement of one of the covers in trilayer structures decreases the SRT thickness. In particular, for Mo underlayer the magnetization rotates towards film plane when Co thickness exceeds value of $1.1 \mathrm{~nm}[6]$. Therefore for Co thickness range between $1.1 \mathrm{~nm}$ and $1.9 \mathrm{~nm}$ the magnetization in $\mathrm{Au} / \mathrm{Co} / \mathrm{Au}$ structures is perpendicular to the film plane whereas in $\mathrm{Mo} / \mathrm{Co} / \mathrm{Au}$ trilayers is aligned in the sample plane. The comparison of magnetic hysteresis loops recorded for the above two configurations with Co thickness equal to $1.35 \mathrm{~nm}$ is depicted in Fig. 1. The shape of the loop for $\mathrm{Au} / \mathrm{Co} / \mathrm{Au}$ is ideally rectangular. After saturation the magnetization signal is kept unchanged while decreasing applied magnetic field even to zero. As a consequence, remnant magnetization is equal to 1 as for a film with perpendicular magnetization in the absence of external field. In opposite, the recorded hysteresis loop for $\mathrm{Mo} / \mathrm{Co} / \mathrm{Au}$ structure has completely different shape. From saturation state the magnetization decreases linearly below $3.5 \mathrm{kOe}$ taking a zero value in the absence of magnetic field. Such dependence reflects the continuous magnetization rotation from perpendicular orientation. Therefore in equilibrium without magnetic field the magnetization is aligned in the sample plane.
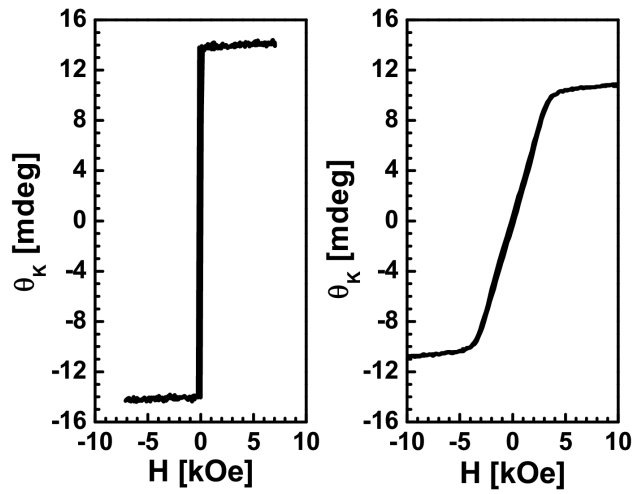

Fig. 1. The magnetic hysteresis loops recorded for $\mathrm{Au} / \mathrm{Co} / \mathrm{Au}$ (left part) and $\mathrm{Mo} / \mathrm{Co} / \mathrm{Au}$ (right part) trilayers for Co layer thickness equal to $1.35 \mathrm{~nm}$.

In order to execute the idea of magnetic anisotropy patterning in ultrathin Co films by the substrate, the appropriate spatially modulated structure of the underlayer is required. As shown in Fig. 1 an island-like growth on Mo buffer is a good candidate for this purpose. The AFM image of morphology of such patterned underlayer is depicted in Fig. 2. It seems that Au island-like growth on Mo is a unique one, because the other combinations (e.g. Au on $\mathrm{V}$ or $\mathrm{Pt}$ on $\mathrm{Mo}$ ) do not exhibit such tendency. Although the $\mathrm{Au}$ islands are formed on Mo in the wide range of substrate temperature between $200^{\circ} \mathrm{C}$ and $850^{\circ} \mathrm{C}$ kept during deposition, the optimum temperature was estimated for $500^{\circ} \mathrm{C}$. At this temperature $\mathrm{Au}$ islands have very regular shapes with well defined edges. Their edges are mostly oriented along one of three directions, suggesting the epitaxial growth. We believe that the whole deposited $\mathrm{Au}$ is gathered in islands and between them the bare Mo surface develops. The underlayer morphology shown in Fig. 2 was recorded for nominal thickness of deposited Au equal to $2.6 \mathrm{~nm}$. In this case the lateral size of $\mathrm{Au}$ islands ranges between $80 \mathrm{~nm}$ and $300 \mathrm{~nm}$ whereas their height oscillates about $23 \mathrm{~nm}$. It is easy to realize that these parameters are dependent on the amount of deposited material. Therefore the lateral size and the height of $\mathrm{Au}$ islands as well as the distance between them are straightforwardly tunable.
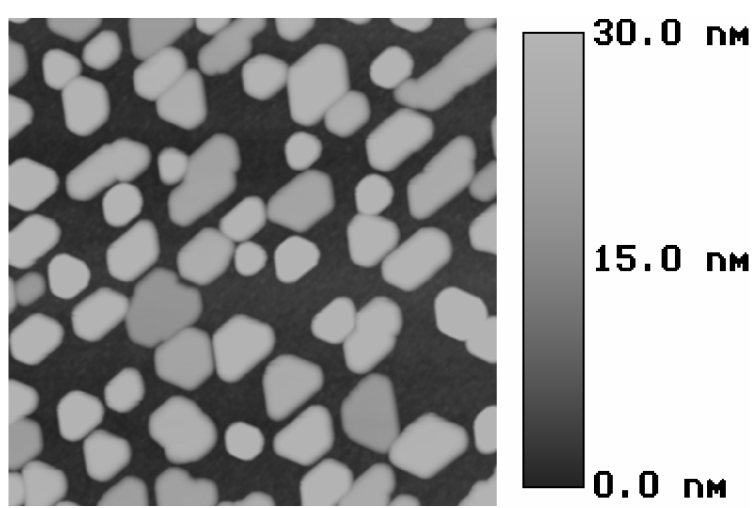

Fig. 2. The AFM image (scan size $3 \times 3 \mu \mathrm{m}$ ) of Au islands grown upon deposition of $2.6 \mathrm{~nm} \mathrm{Au}$ on Mo buffer at $500^{\circ} \mathrm{C}$.

Consequently, it is expected that upon deposition of Co layer on patterned underlayer as shown in Fig. 2 and then of $\mathrm{Au}$ overlayer, the array of magnetic dots should occur. Combining two completely different magnetization reversal processes for $\mathrm{Mo} / \mathrm{Co} / \mathrm{Au}$ and $\mathrm{Au} / \mathrm{Co} / \mathrm{Au}$ trilayers shown in Fig. 1 with morphology of underlayer depicted in Fig. 2, it is easy to anticipate that magnetization of Co layer deposited on $\mathrm{Au}$ islands is oriented in perpendicular direction to the sample plane whereas between islands, where the Co layer is adjacent to Mo surface, magnetization is aligned in the plane. Thus the dimensions of magnetic dots fabricated in such way accurately correspond to the dimensions of $\mathrm{Au}$ islands on Mo buffer. This scenario is obviously valid only for Co thickness range between $1.1 \mathrm{~nm}$ and $1.9 \mathrm{~nm}$ [6]. The example of hysteresis loop recorded for nominal thickness of deposited $\mathrm{Au}$ underlayer equal to $1.8 \mathrm{~nm}$ and the thickness of Co layer equal to $1.6 \mathrm{~nm}$ is shown in Fig. 3. For the external field much higher than saturating one the Co layer both on $\mathrm{Au}$ islands and between them is magnetized in perpendicular direction to the film plane. 
When its value decreases below $6 \mathrm{kOe}$ the magnetic signal begins to decrease linearly with magnetic field. In this stage of reversal process the magnetization of Co layer grown on Mo rotates towards in-plane orientation, whereas the magnetization of Co deposited on $\mathrm{Au}$ islands is unaffected, e.g. remains perpendicular to the sample plane. It is worth noticing that even in the absence of external field the remnant magnetization although lower than 1 takes non-zero value. It is very clearly visible that hysteresis shown in Fig. 3 is composed of two radically different in shape loops, which are shown in Fig. 1 for $\mathrm{Au} / \mathrm{Co} / \mathrm{Au}$ and $\mathrm{Mo} / \mathrm{Co} / \mathrm{Au}$ systems. Moreover, the surface coverage ratio by $\mathrm{Au}$ islands (21\%) observed by AFM for the coverage of $\mathrm{Au}$ equal to $1.8 \mathrm{~nm}$ excellently corresponds to the contribution $(23 \%)$ of rectangular part of the hysteresis loop in the total magnetic signal measured at saturation state. Because the diameter of the laser beam used in magnetooptical measurements is several orders of magnitude larger than the lateral size of $\mathrm{Au}$ islands and thus of magnetic dots as well as the distance between the dots, the recorded signal is averaged over relatively large area and in consequence it is the sum of two different signals coming from two magnetically different phases of the studied structure. This observation proves indirectly the existence of the expected magnetic dots structure clearly described above.

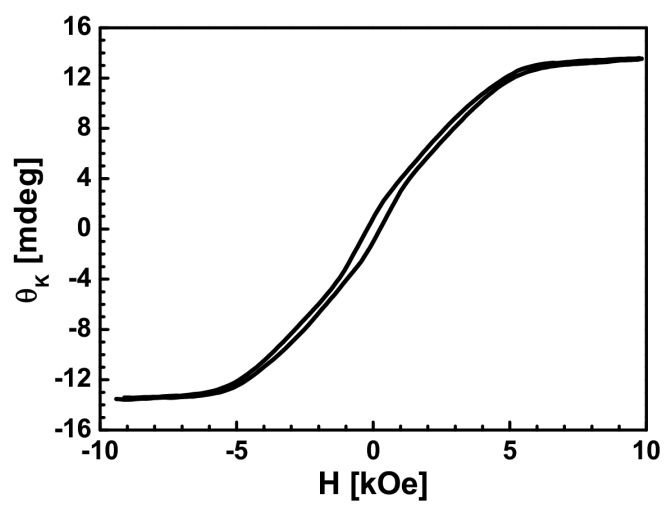

Fig. 3. The hysteresis loop recorded for $1.6 \mathrm{~nm}$ thick Co layer grown on patterned ( $\mathrm{Au}$ islands on $\mathrm{Mo}$ ) buffer and capped with Au layer $8 \mathrm{~nm}$ thick.

\section{Summary}

Combining the considerable dependence of ultrathin Co film anisotropy on its thickness and type of adjacent covers in trilayer systems with spatially patterned underlayer we have revealed the possibility to fabricate an array of magnetic dots several dozen nanometer in size with perpendicular to the film plane magnetization. The unique $\mathrm{Au}$ growth on Mo at elevated temperatures offers the appropriate underlayer structure. The Co layer deposited on $\mathrm{Au}$ islands exhibits perpendicular magnetization to the film plane whereas between islands in the sample plane. Such materials are promising for application as patterned media in high density magnetic storage devices and as the sources of well defined magnetic field in nanometer scale for spintronics.

\section{Acknowledgments}

This work was done under the scientific project N N507 452134 granted by the Ministry of Science and Higher Education in Poland in 2008-2011 and by the Polish National Scientific Network ARTMAG "Magnetic nanostructures for spintronics".

\section{References}

[1] I. Chado, C. Goyhenex, H. Bulou, J.P. Bucher, Phys. Rev. B 69, 085413 (2004).

[2] N. Weiss, T. Cren, M. Epple, S. Rusponi, G. Baudot, S. Rohart, A. Tejeda, V. Repain, S. Rousset, P. Ohresser, F. Scheurer, P. Bencok, H. Brune, Phys. Rev. Lett. 95, 157204 (2005).

[3] C. Chappert, H. Bernas, J. Ferre, V. Kottler, J.P. Jamet, Y. Chen, E. Cambril, T. Devolder, F. Rousseaux, V. Mathet, H. Launois, Science 280, 1919 (1998).

[4] S.P. Li, W.S. Lew, J.A.C. Bland, L. Lopez-Diaz, C.A.F. Vaz, M. Natali, Y. Chen, Phys. Rev. Lett. 88, 087202 (2002).

[5] M. Kisielewski, A. Maziewski, M. Tekielak, A. Wawro, L.T. Baczewski, Phys. Rev. Lett. 89, 087203 (2002).

[6] A. Maziewski, M. Kisielewski, Z. Kurant, M. Tekielak, A. Wawro, L.T. Baczewski, Mol. Phys. Rep. 40, 119 (2004). 